\title{
Elements of Structural Masonry Reinforced with Sisal
}

\section{Fibers}

Indara Soto Izquierdo and Marcio Antonio Ramalho

Department of Structural Engineering, University of São Paulo, São Paulo 13566538, Brazil

\begin{abstract}
There is great interest in the use of natural fibers as reinforcement to obtain new construction materials due to its low cost, high availability and reduced energy consumption for its production. This paper evaluates the incorporation of sisal fibers of $20 \mathrm{~mm}$ and $40 \mathrm{~mm}$ in length and volume fraction of $0.5 \%$ and $1 \%$ for concrete masonry structural blocks, and determines the use of these units to build prisms and mini-walls. Laboratory tests were carried out to characterize the physical of blocks and mortar, in addition to the axial compression tests of the units, prisms, and mini-walls. The sisal had low apparent density and high water absorption, which is a common feature of such material due to the high incidence of permeable pores. The physical properties of the blocks with and without addition complied with the standard requirements established to validate their use. The obtained results showed that the fiber-reinforced mini-walls obtained values very close to or even higher than those obtained for the mini-walls without fibers, demonstrating better performance than the blocks and prisms.
\end{abstract}

Key words: Composites, sisal fiber, concrete block, compressive strength.

\section{Introduction}

Tropical countries like Brazil have an abundance and wide diversity of cultivated vegetables that provide fibers, which enable applying such elements into the construction industry, improving the physical and mechanical characteristics and yielding a better structural performance.

This research focuses on the fundamental scientific application of new structural materials for sustainable constructions, called "green materials". The vegetable fiber composite elements represent a good example of materials aforementioned.

The purpose of this work is to produce structural concrete blocks with and without the addition of sisal fibers and to conduct a comparative study of the mechanical behaviour of the blocks, prisms and mini-walls with and without the addition of sisal fibers by axial compression tests.

Corresponding author: Indara Soto Izquierdo, M.Sc., engineer, research fields: structural analysis, structure of concrete and structural masonry. E-mail: indara@sc.usp.br.

\section{Experimental Program}

A detailed characterization of the materials was performed as well as axial compression tests of the blocks, prisms and mini-walls with and without added fibers. The testing equipment used belongs to the Structures Laboratory of EESC (School of Engineering in São Carlos), USP (University of São Paulo).

\subsection{Fiber}

The production of composites used sisal fibers provided by the company Sisal Weaving located in the state of Bahia, Brazil. The sisal fiber was the impregnated mineral oil emulsion. This type of fiber is generally used to make Bale Twine wires (wires for agricultural exports). It is characterized as dry fibers, brushed and in a good state of maturation, with brightness and maximum humidity of $13.5 \%$. The treatment with mineral oil fibers can decrease their high capacity to absorb water, protect them against alkaline aggression and reduce impurities and residual dust (Fig. 1). 


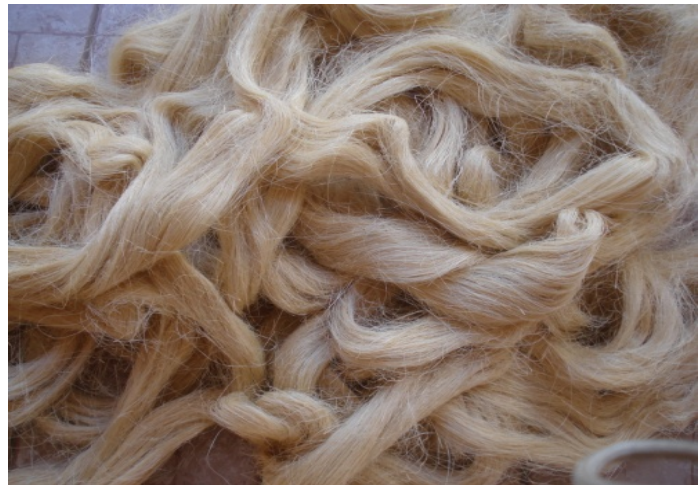

Fig. 1 Sisal fiber.

\subsection{Blocks}

The concrete blocks were manufactured with characteristic strength of $4 \mathrm{MPa}$ at Tatu Premolded Company in Limeira, Sao Paulo, Brazil. The sisal fibers were placed directly into the mixer of the machine that makes the blocks. The difference between series was by fiber content added to the concrete volume and length. Table 1 shows the nomenclature adopted for the building blocks of each series.

The blocks were characterized by means of medium strength $\left(f_{b m}\right)$ and characteristic strength $\left(f_{b k}\right)$, according to NBR (Brazilian Norm) [1]. The test was carried out with a press brand Ele and Autotest 2000 model, equipped with two plates used as support. The machine has a 2,000 kN capacity and load speed imposition. The blocks were tested in dry air as shown in Fig. 2.

\subsection{Prisms}

According to NBR [2], two blocks of prisms were adopted as the elements that can be used to estimate the compressive strength of masonry walls for structural concrete hollow blocks. A total of 60 prisms were tested (12 for each series) with total dimensions of $14 \mathrm{~cm} \times 19 \mathrm{~cm} \times 39 \mathrm{~cm}$ (thickness $\times$ width $\times$ height), and a $1 \mathrm{~cm}$ thick mortar joint.

The prisms were tested in a servo-controlled INSTRON machine model 8506 which used a SYSTEM 5000 for data acquisition. The plates (35 $\mathrm{mm}$ of thickness) was located in the head of the specimen to ensure a uniform load distribution as shown in Fig. 3. Ceiling tiles were used as capping material at both ends of the prism.

\subsection{Mini-walls}

Mini-walls are elements that can be used to estimate the strength of masonry walls. Three mini-walls were built with blocks of each series totalling 15 elements

Table 1 Block series produced.

\begin{tabular}{lll}
\hline $\begin{array}{l}\text { Block structural } \\
\text { nomenclature }\end{array}$ & $\begin{array}{l}\text { Volume fraction of } \\
\text { added fibers (\%) }\end{array}$ & $\begin{array}{l}\text { Fiber length } \\
\text { (mm) }\end{array}$ \\
\hline BE (reference blocks) & - & - \\
BE 20\%-0.5\% & 0.5 & 20 \\
BE 40\%-0.5\% & 0.5 & 40 \\
BE 20\%-1\% & 1 & 20 \\
BE 40\%-1\% & 1 & 40 \\
\hline
\end{tabular}

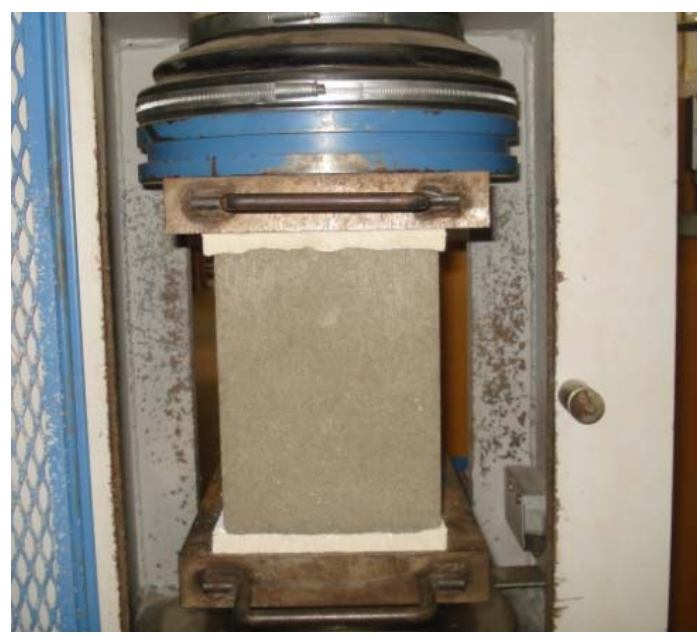

Fig. 2 Test of resistance to compression of the blocks.

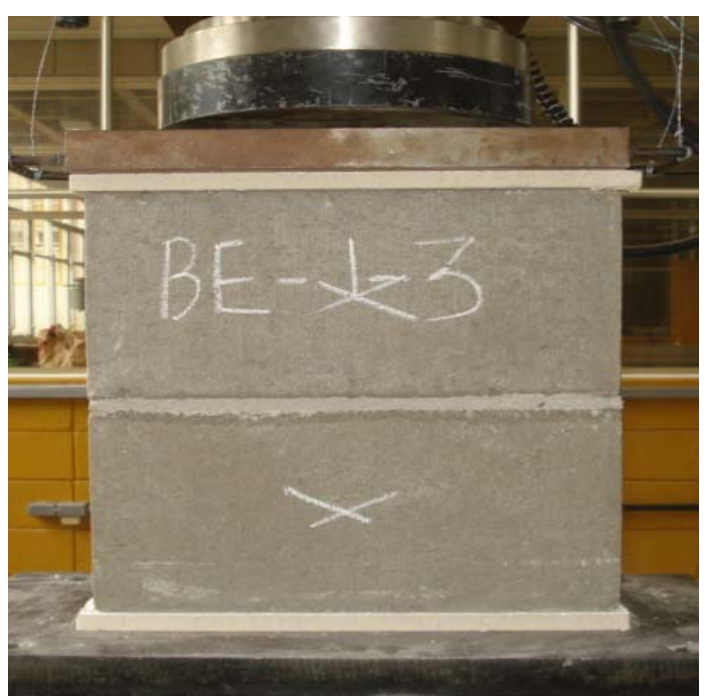

Fig. 3 Testing of compressive strength of prisms. 
to be tested. There were five rows, resulting in eight blocks and four half-blocks. The mini-walls overall dimensions were $14 \mathrm{~cm} \times 79 \mathrm{~cm} \times 99 \mathrm{~cm}$ (thickness $\times$ width $\times$ height), with vertical and horizontal mortar joints as shown in Fig. 4.

The preparation of prisms and small walls used only one type of mortar. The mortar is mixed type (of cement, lime and sand) which is the most suitable for use in masonry structures. The British Standard [3] prescribes traces of medium and low resistance. The mortar was chosen medium resistance, whose trace volume is 1:0.5:4.5.

The tests were performed on the already mentioned servo-controlled INSTRON machine. One steel profile on top of the wall assured uniform load distribution on the body of the test as shown in Fig. 5 .

\section{Experimental Results}

The following are the results and analysis of the facts observed in the tests corresponding to the experimental program.

\subsection{Fiber}

Table 2 shows the test values of the physical characterization of the fibers.

The low apparent density and high water absorption are common characteristics of vegetable fibers by a high incidence of permeable pores that include gaps and lumens. Savastano [4] obtained apparent density values between $400 \mathrm{~kg} / \mathrm{m}^{3}$ and $500 \mathrm{~kg} / \mathrm{m}^{3}$, lower than those obtained for the fibers used in this work. The maximum absorption values in $24 \mathrm{~h}$ were lower than those obtained by Filho [5] and Savastano [4], 193\% and $151 \%$, respectively. The reason for this may be related to the treatment based on mineral oil that impregnated the fibers, producing a smaller amount of pores.

\subsection{Blocks}

Table 3 shows a summary of the physical properties of five types of concrete blocks used.

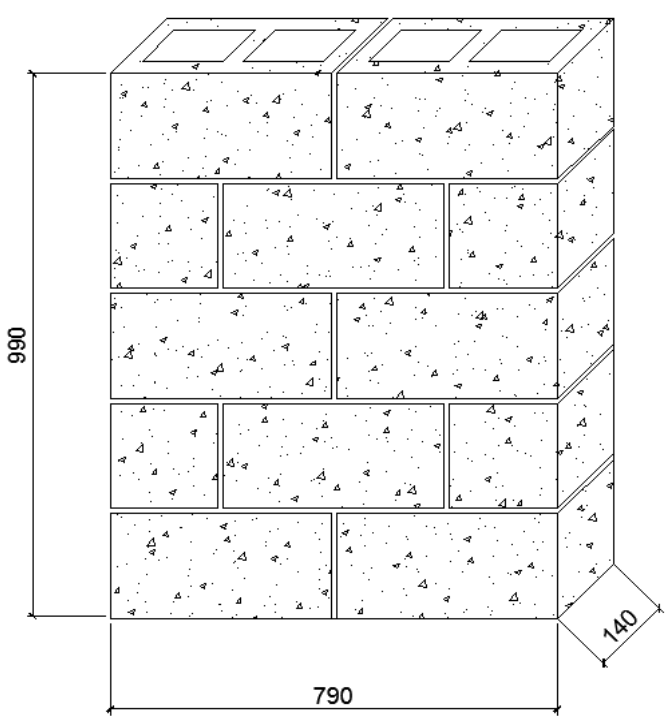

Fig. 4 Dimensions of the walls (mm).

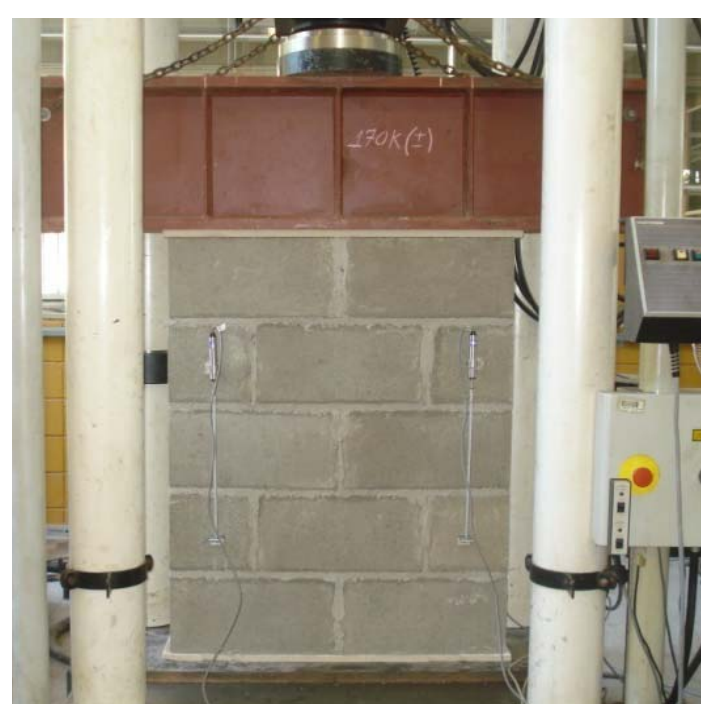

Fig. 5 Test of resistance to compression of the mini-walls.

Table 2 Physical characterization of fibers.

\begin{tabular}{lllll}
\hline Values & $\begin{array}{l}\text { Real } \\
\text { density } \\
\left(\mathrm{kg} / \mathrm{m}^{3}\right)\end{array}$ & $\begin{array}{l}\text { Apparent } \\
\text { density } \\
\left(\mathrm{kg} / \mathrm{m}^{3}\right)\end{array}$ & $\begin{array}{l}\text { Humidity } \\
\text { content } \\
(\%)\end{array}$ & $\begin{array}{l}\text { Water } \\
\text { absorption } \\
24 \mathrm{~h} \mathrm{( \% )}\end{array}$ \\
\hline Mean & 970.62 & 740.22 & 10.47 & 139 \\
\hline
\end{tabular}

Table 3 Physical characteristics of the blocks.

\begin{tabular}{lcll}
\hline Block type & $\begin{array}{l}\text { Water } \\
\text { absorption }(\%)\end{array}$ & $\begin{array}{l}\text { Net area } \\
\left(\mathrm{mm}^{2}\right)\end{array}$ & $\begin{array}{l}\text { Dry air density } \\
\left(\mathrm{g} / \mathrm{cm}^{3}\right)\end{array}$ \\
\hline BE & 7.78 & $29,214.11$ & 2.18 \\
BE 20-0.5\% & 10.17 & $29,156.92$ & 2.12 \\
BE 40-0.5\% & 10.61 & $29,123.37$ & 2.08 \\
BE 20-1\% & 10.00 & $29,381.81$ & 2.11 \\
BE 40-1\% & 8.91 & $28,791.12$ & 2.18 \\
\hline
\end{tabular}


The standard NBR [6] establishes that the water absorption for concrete structural blocks must be less than or equal to $10 \%$. The presence of fibers in the concrete caused a greater absorption of the units, indicating a higher incidence of permeable pores. However, the values obtained for some units with fibers are only slightly larger than the threshold, which can be considered acceptable. The fibers also caused the dry air density values to be smaller for the reinforced blocks, when compared with the reference blocks, due to the gaps introduced into them, acting as air incorporators. These effects were not as significant in blocks with higher content and size of fibers. The moulding of these blocks was conducted in increased feeding times, vibration and shaking of the machine on account of the higher fiber amounts. Consequently, the voids and the entrained air were reduced.

All units had very similar net area values, since they were produced on the same machine with the same mould.

3.3 Compressive Strength of the Blocks with and without Fiber Addition

Table 4 shows the mean compressive strength values and characteristic strength of each type of block. These results were calculated using the gross area of the units. The medium strength $\left(f_{b m}\right)$ and characteristic strength $\left(f_{b k}\right)$ were calculated according to NBR [6]. The table also shows the SD (standard deviation) of the values. Fig. 6 enables to better understand the results.

All units had characteristic compressive strength values and were placed in the $4 \mathrm{MPa}$ structural blocks class, as requested by the manufacturer. As can be seen, the blocks with fibers showed a decrease in compressive strength by $41 \%$ in average when compared to the reference blocks. It may be that the presence of sisal in the concrete caused failures in the material, lower compactness and most voids. This can be evidenced by lower densities of the blocks with fibers when compared to the reference blocks.
3.4 Compressive Strength of Prisms with and without Fiber Addition

Table 5 shows the results of compressive strength corresponding to the gross area of the prisms. Efficiency factors of prism/block $\left(f_{p} / f_{b m}\right)$ are also shown. Compressive mean strength of prism is $f_{p}$ and of block is $f_{b m}$.

With respect to the efficiency factor $\left(f_{p} / f_{b m}\right)$, the overall average value obtained was of 0.54 , slightly lower than expected when considering the resistance of the blocks used.

The fiber-reinforced prisms showed a decrease average resistance of $28 \%$ compared to the prisms without fibers, as can be seen in Fig. 7 .

3.5 Compressive Strength of Mini-walls with and without Fiber Addition

The compressive strength results for the mini-walls

Table 4 Compressive average strength and characteristic strength of blocks.

\begin{tabular}{llll}
\hline Block type & $\begin{array}{l}\text { Average } \\
\text { resistance }\left(f_{b m}\right) \\
(\mathrm{MPa})\end{array}$ & $\begin{array}{l}\text { Standard } \\
\text { deviation } \\
(\mathrm{MPa})\end{array}$ & $\begin{array}{l}\text { Characteristic } \\
\text { strength }\left(f_{b k}\right) \\
(\mathrm{MPa})\end{array}$ \\
\hline BE & 11.26 & 1.06 & 9.43 \\
BE 20-0.5\% & 7.11 & 0.48 & 6.25 \\
BE 40-0.5\% & 6.00 & 0.61 & 4.81 \\
BE 20-1\% & 7.16 & 1.22 & 6.10 \\
BE 40-1\% & 6.43 & 0.78 & 5.22 \\
\hline
\end{tabular}

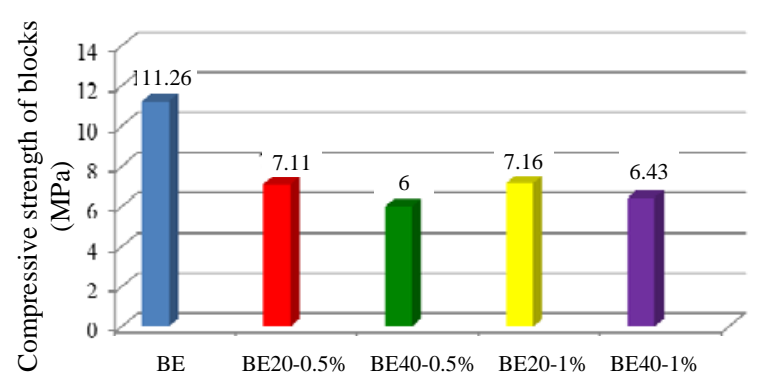

Fig. 6 Average resistance to compression of the blocks.

Table 5 Compressive strength of prisms.

\begin{tabular}{lll}
\hline Prism type & $\begin{array}{l}\text { Average resistance }\left(f_{p}\right) \\
(\mathrm{MPa})\end{array}$ & Efficiency $\left(f_{p} / f_{b m}\right)$ \\
\hline PR & 5.19 & 0.46 \\
PR 20-0.5\% & 3.49 & 0.49 \\
PR 40-0.5\% & 3.25 & 0.54 \\
PR 20-1\% & 4.37 & 0.61 \\
PR 40-1\% & 3.82 & 0.59 \\
\hline
\end{tabular}




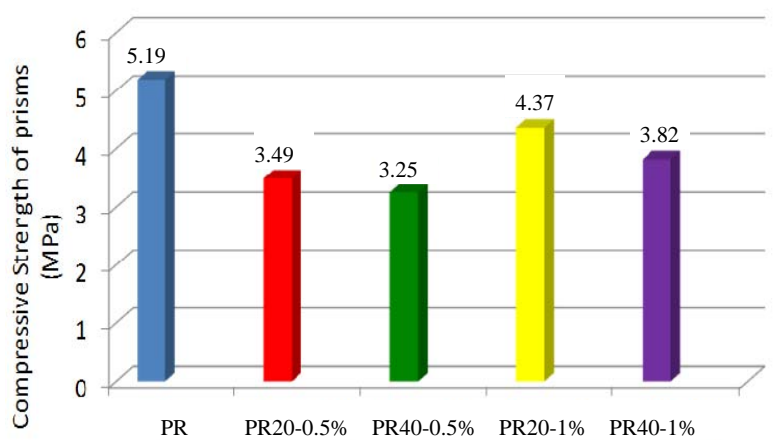

Fig. 7 Compressive strength of prisms.

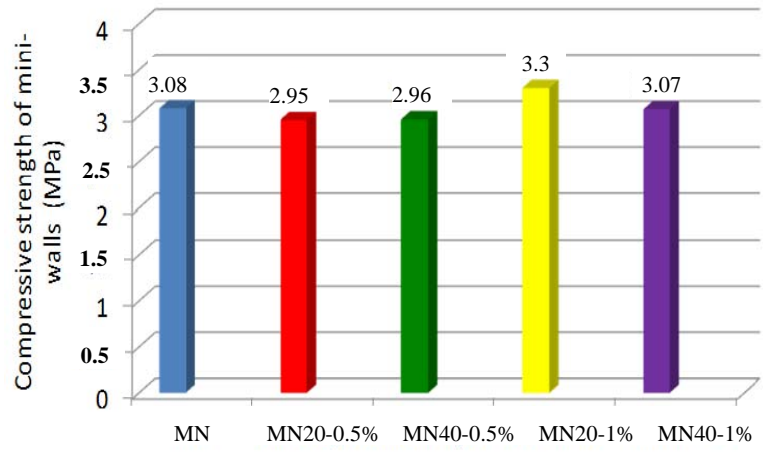

Fig. 8 Compressive strength of mini-walls.

Table 6 Compressive strength of mini-walls.

\begin{tabular}{llll}
\hline Element type & $\begin{array}{l}\text { Average resistance }\left(f_{m p}\right) \\
(\mathrm{MPa})\end{array}$ & $f_{m p} / f_{b k}$ & $f_{m p} / f_{p}$ \\
\hline MN & 3.08 & 0.33 & 0.59 \\
MN 20-0.5\% & 2.95 & 0.47 & 0.81 \\
MN 40-0.5\% & 2.96 & 0.62 & 0.91 \\
MN 20-1\% & 3.30 & 0.54 & 0.76 \\
MN 40-1\% & 3.07 & 0.59 & 0.80 \\
\hline
\end{tabular}

based on gross area are given in Table 6. The efficiency factors of the mini-walls/blocks and mini-walls/prisms are also shown.

It is noted that there are no significant changes in the compressive strength of mini-walls with sisal fibers, resulting in values very close to or even higher than those obtained for the mini-walls without fibers. This can be verified in Fig. 8.

In fact, the element consisting of blocks with the addition of $20 \mathrm{~mm}$ and fiber content of $1 \%$ showed greater strength than the wall without fibers.

\section{Discussion of Test Results}

The analysis reveals that the blocks with fibers showed a decrease in overall average strength equal to $41 \%$, when compared to blocks without fibers. For the prisms, the decrease was of $28 \%$ and for the mini-walls the average reduction was very small, only $3 \%$. It can be considered that this fact is associated with the mechanical behavior, which is different for each type of component or element studied and the degree of development of tensile stresses.

The reinforced units, even when it ruptured, maintained their share by the fibers together, without losing its continuity and making a progressive rupture. In prisms, in most cases the rupture occurred by the development of vertical cracks along the lateral septa. They started close to the mortar joints, which then spread throughout the height of the element, with a characteristic break of traction in the region near the joint. As for the cracking state of the mini-walls, vertical cracks of traction that began in the vertical joints were observed, progressing to cutting the blocks and causing them to break. The appearance of this type of cracks can be explained by the presence of vertical joints in the mini-walls.

The mini-walls are composed of a group of masonry units linked by mortar through horizontal and vertical joints aligned and lagged half a block. This type of specimen is the one that best represents the failure mode of masonry, because this is an element that represents a small portion of the material with all its main features.

In the mini-walls, sisal fibers probably showed better performance because more tensile stresses in the units developed. This is explained by two main reasons. The first is that the specimen which has larger dimension is submitted to a lower confinement produced by the test apparatus. Furthermore, higher traction in the blocks can also be explained by the fact that there are lagged vertical joints, which does not occur in the prisms.

\section{Conclusions}

The main objective of this research was to study the 
incorporation of sisal fibers for the production of concrete structural blocks and to compare the viability of using these units in masonry structural elements.

With respect to the physical properties of the fibers, they showed low apparent density and high water absorption. In fact, these are common characteristics of natural fibers by a high incidence of permeable pores.

The physical properties of five types of concrete blocks, such as water absorption, densities and net area, met the requirements established by the regulatory codes.

The mini-walls are specimens that represent the best way to rupture the masonry, in which higher tensile stresses appear in the units, mainly due to the lagged vertical joints. Under this type of strength, the fibers act as reinforcement and can effectively and efficiently contribute to improve the resistance capacity of the material. That is why the reinforced mini walls with fibers had a decrease in resistance less in relation to the block and prisms reinforced.

In prisms, there are horizontal tensile stresses of less intensity than in the mini-walls, which are produced only by the rigidity difference between the block and mortar. In addition, for the prisms, especially in the blocks, the compression test is always characterized by a certain effect of confinement that contributes to a reduction of the traction units.
Overall, taking into account the length and content by volume, the composite fibers of $20 \mathrm{~mm}$ and $1 \%$ addition showed the best performance for the blocks and prisms as well as for the mini-walls.

\section{Acknowledgments}

The authors would like to thank to the Department of Structural Engineering, School of Engineering of São Carlos-USP, for permission to carry out this work.

\section{References}

[1] NBR 12118: Blocks Hollow of Single Concrete for Masonry-Test Methods, Brazilian Association of Technical Standards, Rio de Janeiro, 2007. (in Portuguese)

[2] NBR 10837: Calculation of Structural Masonry for Concrete Hollow Blocks, Draft Technical Standard, Brazilian Association of Technical Standards, Rio de Janeiro, 1989. (in Portuguese)

[3] BS 5628: Part 1: Code of Practice for Use of Masonry-Structural Use of Unreinforced Masonry, British Standards Institution, London, 1992.

[4] H.J. Savastano, Materials based reinforced cement with vegetable fiber: Recycling of waste for the construction inexpensive, Ph.D. Thesis, Free-Teaching in Civil Engineering School, Polytechnic University of São Paulo, São Paulo, 2000, pp. 7-22. (in Portuguese)

[5] R.D.T. Filho, Composites reinforced with natural fibers: Experimental characterization, Ph.D. Thesis, Department of Civil Engineering, Catholic University of Rio de Janeiro, Rio de Janeiro, 1997, p. 93. (in Portuguese)

[6] NBR 6136: Blocks Hollow of Single Concrete for Masonry Requirements, Brazilian Association of Technical Standards, Rio de Janeiro, 2007. (in Portuguese) 